\title{
MODELING SUBDIFFUSION USING REACTION DIFFUSION SYSTEMS
}

\author{
MARIO S. MOMMER $\S_{\S}$ AND DIRK LEBIEDZ $\ddagger$
}

\begin{abstract}
In many types of media, and in particular within living cells or within their membranes, diffusing species do not follow Fick's laws, but instead show transient subdiffusive behavior. Formulating spatiotemporal models that take this behavior into account is a delicate matter, as one is faced with the choice of resorting either to fractional calculus or to microscopic descriptions. In this article, we provide an alternative designed to be easier to tackle analytically and numerically than the existing approaches. Specifically, starting from the Continuous Time Random Walk model, we construct linear reaction diffusion systems that can be used as components within such a model, and which capture the defining properties of subdiffusion. We show how to impose physically relevant parameters, and prove stability and mass conservation. While applications to cellular biology are our main motivation, our approach is abstract, and should thus be applicable to any situation where anomalous subdiffusion is observed.
\end{abstract}

Key words. Diffusion, Anomalous Diffusion, Subdiffusion, Reaction Diffusion Equations.

AMS subject classifications. 76R50, 35K57.

1. Introduction. In media with nontrivial, disordered microscopic substructures, one observes a special type of anomalous diffusion called subdiffusion [2]. While a particle diffusing according to Fick's laws has a mean square displacement that scales linearly with time,

$$
\left\langle x(t)^{2}\right\rangle \sim K_{1} t
$$

in the case of subdiffusive anomalous diffusion one observes a behavior of the type

$$
\left\langle x(t)^{2}\right\rangle \sim K_{\alpha} t^{\alpha}
$$

with $0<\alpha<1$. (Superdiffusion, a close cousin of subdiffusion, follows (1.1) with $\alpha>1$.) Within living cells, (1.1) is often observed only for limited, although eventually quite long, time scales. For longer time scales, the behavior is normally diffusive [12]. The time scale is determined by the shape and size of the particles.

The reason for subdiffusive behavior within cells is that cells are tightly packed with a variety of organelles and proteins, yielding a somewhat porous, gel-like medium [12]. This phenomenon is called "molecular crowding" in the literature. Indeed, the cytosol (the interior of the cell), which is usually thought of as a watery solution, has about $40 \%$ of its volume taken up by proteins of a vast variety of sizes and shapes. And it is the size and shape distribution of these proteins, and the size and shape of the species under study, that give rise to subdiffusive behavior. And since only a finite range of sizes is present, this subdiffusive behaviour is transient [11].

For the purpose of constructing spatiotemporal models of intracellular dynamics, the importance of subdiffusion lies in the fact that it influences reaction rates [10], and, in our opinion, in the fact that it is non-Markovian. By introducing memory to the mix, it has the potential of significantly enriching the nonlinear dynamics of even simple models. But studying and formulating such models is relatively difficult.

\footnotetext{
${ }^{\ddagger}$ Interdisciplinary Center for Scientific Computing, University of Heidelberg, Im Neuenheimer Feld 368, 69120 Heidelberg, Germany.

$\S$ To whom correspondence should be addressed (e-mail: mario.mommer@iwr.uni-heidelberg.de). Supported by the Center for Modeling and Simulation in the Biosciences (BioMS).
} 
One may use fractional calculus, in the form of the subdiffusive fractional diffusion equation, which has the drawback of not reproducing a return to Fickian diffusion, and of having infinite memory. The only alternative we are aware of are atomistic, particle by particle descriptions. While easy to construct, such models tend to be hard to tackle analytically, and very expensive to simulate numerically for realistic system sizes.

The starting point for a mathematically rigorous understanding of subdiffusion is the Continuous Time Random Walk (CTRW) model, which we briefly review in $\S$ 2. In contrast to the classical random walk model of Brownian motion, in the CTRW particles move and wait in an alternating fashion, and the jump length and waiting time are random variables. Depending on the choice of PDFs, one can obtain a whole spectrum of different diffusive behaviors, including Fickian diffusion, superdiffusion, and subdiffusion.

In $\S 3$ of this article, we propose and derive an additional option, designed to be easier to handle numerically and analytically. We start by introducing a fairly simple modification of the CTRW, which we call the Robotic Continuous Time Random Walk, and where a State appears as an additional random variable. This additional variable allows us to generate a family of different waiting time PDFs from simple exponential (Poisson) distributions. From this we derive a system of reaction diffusion equations where the pseudospecies $u_{k}, k=1, \ldots, N$ (which represent the concentration of particles in state $k$ ) diffuse normally, and where the switching between states is accounted for by a suitable reaction term. The concentration profile of the species that shows subdiffusive behavior is given by the sum of the pseudospecies, $u(t)=$ $\sum_{k=1}^{N} u_{k}(t)$.

The system we obtain from this construction is stable and conserves mass. It also has the desired property of showing return to normal diffusion for long time scales. In $\S 4$ we give a precise recipe to construct such systems with a desired set of natural phenomenological parameters, which are, maximum average diffusion coefficient, essential time of return to Fickian diffusion, and long-term, or minimum, diffusion coefficient. Finally, in $\S 5$, we show how this construction performs in numerical experiments. It is our hope that this tool will generally simplify the inclusion of subdiffusive effects in spatiotemporal models of intracellular dynamics.

2. Anomalous Diffusion. Before developing the reaction diffusion model, we give a brief introduction to the fundamentals of anomalous diffusion, focusing mainly on subdiffusion. The material in this section follows, unless otherwise stated, the account by Klafter and Metzler [8].

Diffusive transport refers to the movement in space of an ensemble of particles of a given species, accomplished by random motions caused by thermodynamic fluctuations. The best known example of diffusive transport is the one caused by Brownian motion and which is described by Fick's laws, leading to the well known parabolic diffusion equation

$$
\frac{\partial u}{\partial t}=\triangle u .
$$

This form of diffusion can be found, for instance, in the diffusion of ink in water. Under Fickian diffusion, the mean square displacement of a particle scales linearly with time; that is,

$$
\left\langle x(t)^{2}\right\rangle \sim K_{1} t .
$$


Any diffusive transport phenomenon which does not follow (2.1) is considered to be anomalous diffusion.

There are, of course, many possible deviations from (2.1), but it is sufficient for the description of many phenomena to consider diffusive transport obeying

$$
\left\langle x(t)^{2}\right\rangle \sim K_{\alpha} t^{\alpha}
$$

for $\alpha>0$, which includes Fickian diffusion as a special case. If $0<\alpha<1$, the diffusion process is called subdiffusive, or subdiffusion, and if $1<\alpha$ it is called superdiffusion.

This type of diffusion can be recovered, for all values of $\alpha$, from the continuous time random walk (CTRW) model which we review briefly in $\S 2.1$. From this model one obtains continuum evolution equations called fractional diffusion equations (FDE), that have the form

$$
\frac{\partial u}{\partial t}={ }_{0} D_{t}^{1-\alpha} K_{\alpha} \triangle u
$$

for subdiffusion $(0<\alpha<1)$, and

$$
\frac{\partial u}{\partial t}=K_{-\infty}^{\alpha} \bar{D}_{x}^{\alpha} u
$$

for superdiffusion $(1<\alpha)$. In $(2.3)$, the operator ${ }_{0} D_{t}^{\alpha}$ is the Riemann-Liouville time derivative, and ${ }_{-\infty} \bar{D}_{x}^{\alpha}$ is the Riesz-Weyl operator. We will not go into further details concerning these operators, or indeed of fractional calculus in general. We note, however, that other fractional differential operators can be used in both cases. See $[5,6]$.

As mentioned in the introduction, our main focus of interest in this article is subdiffusion. Still, we mention superdiffusion a few times, but mostly to put subdiffusive behavior in perspective.

2.1. The Continuous Time - Random Walk (CTRW) model. The continuous time - random walk (CTRW) model is a generalization of the Brownian motion model of diffusion, and was introduced first by Montroll and Weiss in 1965 [9]. In the CTRW, a particle moves (in zero time) a given distance, and then waits for a certain time before moving again. The jump length $\mathbf{r}$, and the waiting time $t$, are random variables drawn from a joint probability density function $\Psi(\mathbf{r}, t)$. For our purposes, it is sufficient to consider only the case where the jump length and the waiting time are independent random variables, so that

$$
\Psi(\mathbf{r}, t)=\lambda(\mathbf{r}) \omega(t)
$$

with $\lambda\left(\mathbf{r}_{1}\right)=\lambda\left(\mathbf{r}_{2}\right)$ whenever $\mathbf{r}_{1}$ and $\mathbf{r}_{2}$ have the same Euclidean norm.

Of particular importance for the characterization of the diffusion process are the characteristic waiting time,

$$
\tau^{*}=\int_{0}^{+\infty} t \omega(t) d t
$$

and the jump-length variance

$$
\Sigma^{2}=\int_{\mathbb{R}^{n}}|r|^{2} \lambda(\mathbf{r}) d \mathbf{r},
$$


each of which may diverge, depending on the situation considered.

If both $\tau^{*}$ and $\Sigma^{2}$ are finite, the long term behavior of the system is described by Fick's laws. A diverging characteristic time with finite jump-length variance gives rise to subdiffusion. In contrast, a finite characteristic time, with divergent jump length variance, gives rise to superdiffusion.

The assumption of independence of waiting time and jump length causes problems in the superdiffusive case, as it allows for arbitrarily long jumps in finite time. Since this assumption of independence is crucial for our construction, we have chosen to avoid superdiffusion.

The subdiffusive behavior we are interested in, i.e., a behavior governed by (2.2), appears when the jump-length has a finite variance, and the waiting-time PDF has the asymptotics

$$
\omega(t) \sim t^{-(1+\alpha)},
$$

with the same $\alpha$.

An important case that will be useful later on is when both $\tau^{*}$ and $\Sigma^{2}$ are finite. In this case, diffusive behavior of the particles can be described by normal diffusion, and the diffusion coefficient is given explicitly by

$$
D_{1}=\frac{\Sigma^{2}}{2 \tau^{*}}
$$

2.2. Time scales. To obtain the fractional diffusion equation from this microscopic model, one considers, for a particular incarnation of the CTRW model, on the propagator $W(x, t)$, which is the PDF that describes the probability for a particle that was at $x_{0}=0, t_{0}=0$ to be at position $x$ at time $t$. Then one considers a series of CTRW models where the PDFs are scaled, as in

$$
\lambda_{h}(\mathbf{r}):=h^{-1} \lambda\left(h^{-1} \mathbf{r}\right), \quad \omega_{s}(t)=s^{-1} \omega(t / s),
$$

and then proceeds to the limit $h \rightarrow 0, s \rightarrow 0$, while keeping $h=K_{\alpha} s^{\alpha}$. One obtains that the limit in distribution of these propagators, which we again denote by $W$, satisfies

$$
\frac{\partial W}{\partial t}={ }_{0} D_{t}^{1-\alpha} K_{\alpha} \triangle W \quad \text { where }(x, t) \in \mathbb{R}^{n} \times(0,+\infty),
$$

with $W(\cdot, 0)=\delta_{0}$ being the Dirac delta at zero. The constant $K_{\alpha}$ is the generalized diffusion coefficient for the subdiffusive fractional diffusion equation. For further details, and for a full account of this derivation we refer to [5].

This passage to the limit has as a consequence that the details of the PDFs $\lambda$ and $\omega$ are removed, and only the convergence or divergence of the jump-length variance and/or the characteristic time, together with the asymptotic behavior in (2.5), play a role. One quickly realizes that an intermediate subdiffusive behavior, with return to normal diffusion for longer time scales, is not within the scope of (2.6).

We will return to the issue of time scales again later, when we have the machinery in place to construct a reaction diffusion model of subdiffusion.

3. Modeling subdiffusion by reaction diffusion systems. Let us first give an outline of our goals. Write $u(x, t): \mathbb{R}^{n} \times(0,+\infty) \rightarrow \mathbb{R}$ for the concentration of a species at the point $x$ at time $t$. Choose some integer $N>1$, and write

$$
u=\sum_{k=1}^{N} u_{i}
$$


with $u_{1}, u_{2}, \ldots, u_{N}: \mathbb{R}^{n} \times(0,+\infty) \rightarrow \mathbb{R}$. We will call the $u_{i}, i=1,2, \ldots, N$ pseudospecies. We now think about the evolution of a parabolic system of the form

$$
\frac{\partial \mathbf{u}}{\partial t}=D \triangle \mathbf{u}+A \mathbf{u}
$$

where $D$ and $A$ are real $N \times N$ matrices, and $D$ is diagonal. Our plan is to construct these matrices in such a way that $u=\sum_{k=1}^{N} u_{i}$ diffuses anomalously, at least within a given range of time scales. This means that, if we observe the behavior of

$$
\xi(t)=\frac{\left\langle x(t)^{2}\right\rangle}{t^{\alpha}}
$$

with an adequate initial condition, we should see a flat region for values of $t$ within that range of time scales.

To construct the matrices $D$ and $A$ needed in (3.1), we will start by modifying the CTRW model slightly, which we do in the next subsection. Then, we will approximate the waiting time PDF by exponentials using the techniques from [1].

3.1. The Robotic Continuous Time Random Walk (RCTRW) model. Suppose that instead of opaque particles moving randomly, we had robots. These robots move by repeating the following steps over and over.

1. Draw at random a number $k \in\{1, \ldots, N\}$ with $P(k=n)=w_{n}$.

2. Wait, drawing the waiting time from the $\operatorname{PDF} \omega_{k}$.

3. Move, drawing the distance and direction from the jump-length $\operatorname{PDF} \lambda_{k}(\mathbf{r})$.

The movement of the robots in this model is the same as in the CTRW with PDFs

$$
\lambda=\sum_{k=1}^{N} w_{k} \lambda_{k} \quad \text { and } \quad \omega=\sum_{k=1}^{N} w_{k} \omega_{k} .
$$

It does, however, add some flexibility which will turn out to be crucial, as it allows us to approximate the PDFs $\lambda$ and $\omega$ by simpler PDFs, and thus move some of the difficulties to an additional variable: the state of the robot. And indeed, the pseudospecies in (3.1) will represent robots in the corresponding state.

3.2. From the RCTRW to a reaction diffusion system. Suppose we have a jump-length PDF $\lambda$ with variance $\Sigma^{2}$, and a waiting time PDF that can be expressed as

$$
\omega(t)=\sum_{k=1}^{N} w_{k} \frac{e^{-t / \tau_{k}}}{\tau_{k}}=\sum_{k=1}^{N} w_{k} \omega_{k}(t),
$$

where of course $\sum_{k=1}^{N} w_{k}=1$. Let us set up the RCTRW with jump-length PDFs $\lambda_{k}=\lambda$, and waiting time PDFs $\omega_{k}$.

Note that the average time a robot spends in a given state $i \in\{1,2, \ldots, N\}$ is $\tau_{i}$. It may then change to state $j$ with probability $w_{j}$. It follows that robots change from state $i$ to state $j$ at the average rate

$$
c_{i j}=\frac{w_{j}}{\tau_{i}} .
$$

We now make the following model assumption. 
Assumption 3.1. As robots in state $i$ move with a jump-length variance $\Sigma^{2}$ and a characteristic waiting time $\tau_{k}$, that movement follows Fick's laws for all time scales.

Form this assumption we obtain the diffusion constant for robots in state $i$,

$$
D_{i}=\frac{\Sigma^{2}}{2 \tau_{i}}
$$

Thus, given an expression of the form (3.2) we can fill the blanks in the system (3.1). The matrix $D \in \mathbb{R}^{N \times N}$ is given by

$$
D=\operatorname{diag}\left(D_{1}, \ldots, D_{N}\right)
$$

and the matrix $A \in \mathbb{R}^{N \times N}$ takes entries

$$
\begin{aligned}
& a_{i j}=c_{j i} \quad \text { for } i \neq j \\
& a_{i i}=-\sum_{k=1, k \neq i}^{N} a_{k i} .
\end{aligned}
$$

Assumption 3.1 is necessary because particles moving with appropriate PDFs can only be described safely by Fick's laws for long time scales (with respect to the corresponding characteristic time), while we use this description also for small time scales. It is not clear to us how large or small this error is, but based on the encouraging numerical results, we conjecture that it is small.

3.3. Approximating the subdiffusive PDF. We now turn to the problem of finding $w_{k}$ and $\tau_{k}, k=1,2, \ldots, N$, such that

$$
\omega(t)=\sum_{k=1}^{N} w_{k} \frac{e^{-t / \tau_{k}}}{\tau_{k}}=\sum_{k=1}^{N} w_{k} \omega_{k}(t)
$$

produces subdiffusive behavior for a range of time scales. To that end, we will try to arrange things in such a way that

$$
\left|\frac{1}{t^{\alpha+1}}-\sum_{k=1}^{N} C w_{k} \frac{e^{-t / \tau_{k}}}{\tau_{k}}\right|<\frac{\epsilon}{t^{\alpha+1}}
$$

for some $\epsilon>0$, and for all $t$ in some interval $I_{\tau}=[a, b] \subset(0,+\infty)$. The normalization factor $C>0$ is used to impose $\int_{0}^{+\infty} \omega(t) d t=1$.

Fortunately, it is known how to produce this type of approximation for arbitrary $\epsilon>0$ and any finite $I_{t} \subset(0,+\infty)$. We refer to [1] where approximation by exponentials is developed for a large class of functions. From there we learn that (3.7) is a special case, as

$$
\frac{1}{t^{\alpha+1}}=\frac{1}{\Gamma(\alpha+1)} \int_{-\infty}^{+\infty} e^{-t e^{s}+(\alpha+1) s} d s .
$$

For a given $t$, the integrand decays rather fast. As a consequence, to compute approximately $t^{-(1+\alpha)}$ using (3.8), we can use quadrature on an appropriate interval of integration $[P, Q]$. Furthermore, if the interval is long enough, and the quadrature accurate enough, we can use (3.8) to produce an approximation of the form (3.7) for a whole range of $t$. It is possible to achieve this with good accuracy on rather large 
intervals with a remarkably low number of terms. In fact, in [1], p. 41, it is proved that

TheOREM 3.2. For any $\beta>0,0<\delta \leq 1$, and $0<\epsilon \leq \min \left\{\frac{1}{2}, \frac{4}{\beta}\right\}$, there exist positive numbers $\tau_{m}$ and $w_{m}$ such that

$$
\left|r^{-\beta}-\sum_{m=1}^{M} w_{m} \frac{e^{-\tau_{m} t}}{\tau_{m}}\right| \leq r^{-\beta} \epsilon \quad \text { for all } \delta \leq r \leq 1 .
$$

For fixed $\beta$ and $\epsilon$, it holds $M=\mathcal{O}\left(\log \delta^{-1}\right)$.

It should be mentioned that in [1] rather precise upper bounds for $M$ are given which have been omitted here.

These approximations are obtained by computing suitable bounds of integration $P<Q$, and then approximating the integral on the right of (3.8) using a summed trapezoidal rule on an equidistant grid. In a departure of [1], we use a summed midpoint rule instead, as all knots have then the same weight. Recall also that the midpoint rule has the same order of accuracy with a slightly more favorable constant.

We add that to obtain an approximation on $I_{t}=[a, b]$ from theorem 3.2 , it is enough to take an approximation on $I_{t}=\left[\frac{a}{b}, 1\right]$ and perform a change of variable.

The next question is, of course, how to choose $\epsilon$ and $I_{t}$. It will turn out that, to impose physically relevant parameters on the system, it is more convenient to specify the interval of integration $[P, Q]$. Furthermore, it is observed that for purposes of achieving subdiffusive behavior, relatively few terms, and thus a rather coarse approximation, is enough. Full implications of the accuracy of the approximation shall be studied elsewhere.

Having chosen $[P, Q]$, and the number of knots $N$ (which coincides with the number of pseudospecies) we write $h=(Q-P) / N$, define the quadrature knots $x_{k}:=(k-1) h+h / 2+P$, and obtain the approximation

$$
\frac{1}{t^{1+\alpha}} \approx \sum_{k=1}^{N} C w_{k} \frac{e^{-t / \tau_{k}}}{\tau_{k}}
$$

with

$$
\tau_{k}=e^{-x_{k}} \quad \text { and } \quad C w_{k}=\frac{h e^{\alpha x_{k}}}{\Gamma(1+\alpha)}
$$

It is easy to check that the $w_{k}$ needed for (3.6) are given by

$$
w_{k}=\frac{e^{\alpha x_{k}}}{\sum_{j=1}^{N} e^{\alpha x_{j}}}=\frac{e^{\alpha h k}}{\sum_{j=1}^{N} e^{\alpha h j}}
$$

where we have used that they must satisfy $\sum_{k=1}^{N} w_{k}=1$.

The question on how to choose $P$ and $Q$ can only be answered after gaining some additional understanding of the properties of the system (3.1).

3.4. The Reaction Diffusion System. Here, we give a characterization of the behavior of the reaction diffusion system (3.1). We assume that an interval $[P, Q]$ has been chosen, and that the $w_{k}, \tau_{k}, k=1,2, \ldots, N$ have been computed as above. We establish existence and uniqueness of the solution, long term behavior, and stability of (3.1). We start by proving some simple results on the reaction matrix $A$. 
Lemma 3.3. Let $A$ be as in (3.4). Then all eigenvalues of $A$ are either zero or have negative real part.

The proof is a straight-forward application of Gerschgorin's Circle Theorem (see for instance [4], p. 320) to (3.4), and is thus omitted.

Lemma 3.4. The dimension of the kernel of the matrix $A$ is 1 . This kernel is spanned by the vector $\mathbf{s}=\left(s_{k}\right) \in \mathbb{R}^{N}$ with entries

$$
s_{k}=\frac{e^{h(1-\alpha)(N-k+1)}}{\sum_{k=1}^{N} e^{h(1-\alpha) k}} .
$$

Here, $h$ is the width of the quadrature mesh as used above. The normalization of $s_{k}$ was chosen in such a way that $\sum_{k=1}^{N} s_{k}=1$, a detail that will be useful later on.

Proof. We start by writing $v^{T}:=(1,1, \ldots, 1) \in \mathbb{R}^{N}$, and observe that $v^{T} A=0$. It follows that the kernel of $A$ is nontrivial.

Let us now rewrite $A$ in the following way. Let

$$
v_{\tau}^{T}=\left(\tau_{1}^{-1}, \tau_{2}^{-1}, \ldots \tau_{N}^{-1}\right) \quad \text { and } \quad v_{w}^{T}=\left(w_{1}, w_{2}, \ldots, w_{N}\right) .
$$

Then $A=F+K$, with $K=v_{\tau} v_{w}^{T}$, and $F=\operatorname{diag}\left(f_{1}, f_{2}, \ldots, f_{N}\right)$ with $f_{k}=-\sum_{k=1}^{N} \frac{w_{k}}{\tau_{k}}$.

The matrix $K$ has rank one, and it is easy to compute its single nontrivial eigenvalue and eigenvector pair $\lambda^{0} \in \mathbb{R}$ and $v^{0} \in \mathbb{R}^{N}$. Let $\left\{u_{1}, u_{2}, \ldots, u_{N}\right\}$ be a basis of $\mathbb{R}^{N}$ such that $u_{1}=v^{0}$ and $\left\{u_{2}, u_{3}, \ldots, u_{N}\right\}$ is a basis for the kernel of $K$. Now, if $u \in \mathbb{R}^{N}$ is such that $A u=0$, we write $u=\sum_{k=1}^{N} \alpha_{k} u_{k}$, and observe that

$$
A u=(F+K) u=D u+\alpha_{1} \lambda^{0} u_{1}=0,
$$

from where we conclude that

$$
u=\alpha_{1} F^{-1} u_{1} .
$$

This proves that the kernel of $A$ has dimension one.

To prove that $\mathbf{s}$ is in this kernel, we start by noting that $A=c A^{0}$, with $A^{0}=\left(a_{i j}^{0}\right)$ given by

$$
\begin{array}{ll}
a_{i j}^{0}=e^{h(\alpha i+j)} & \text { if } i \neq j, \text { and } \\
a_{i i}^{0}=-\sum_{j=1, j \neq i}^{N} e^{h(\alpha j+i)} . &
\end{array}
$$

Thus, $A \mathbf{s}=A^{0} \mathbf{s}=0$ if for each $i$,

$$
\sum_{j=1, j \neq i}^{N} s_{j} e^{h(\alpha i+j)}-\sum_{j=1, j \neq i}^{N} s_{i} e^{h(\alpha j+i)}=0,
$$

which, dropping the normalization in the definition of $s$, reduces to showing that

$$
\sum_{j=1, j \neq i} e^{h[(1-\alpha)(N-j+1)+\alpha i+j]}-e^{h[(1-\alpha)(N-i+1)+\alpha j+i]}=0 .
$$

And in fact, every term of this sum is zero. 
Using lemmas 3.3 and 3.4 we can start looking at dynamic properties of the system (3.1). To this end we consider the initial value problem,

$$
\begin{aligned}
y^{\prime} & =A y \quad \text { with } y:[0,+\infty) \rightarrow \mathbb{R}^{N}, \\
y(0) & =y^{0}=\left(y_{k}^{0}\right) \in \mathbb{R}^{N} .
\end{aligned}
$$

LEMmA 3.5. A solution $y(t)=\left(y_{1}(t), y_{2}(t), \ldots, y_{N}(t)\right)$ of (3.11) satisfies

$$
\begin{array}{r}
\left(\sum_{k=1}^{N} y_{k}(t)\right)^{\prime}=0 . \\
\lim _{t \rightarrow+\infty} y(t)=\left(\sum_{k=1}^{N} y_{k}^{0}\right) \mathbf{s}
\end{array}
$$

Proof. The proof of (3.13) follows from $y^{\prime}=A y$ and the fact that $\sum_{k=1}^{N} a_{k i}=0$ for each $i$. Assertion (3.14) follows from (3.13), and lemmas 3.3 and 3.4.

Next, we are interested in some basic properties of our reaction diffusion system on a bounded domain. Specifically, we are interested in the stability of the system and its long term behavior, as well as in conservation of mass.

THEOREM 3.6. Let $\Omega \in \mathbb{R}^{n}$ be a bounded, simply connected domain with Lipschitz boundary, and consider the problem, find $u \in C\left((0,+\infty),\left[H^{1}(\Omega)\right]^{N}\right)$ such that

$$
\begin{array}{rlrl}
\frac{\partial \mathbf{u}}{\partial t} & =D \Delta \mathbf{u}+A \mathbf{u} & & \text { on } \Omega \times(0,+\infty) \\
\mathbf{u}(\cdot, 0) & =\mathbf{u}^{0}, & \\
\frac{\partial \mathbf{u}}{\partial \mathbf{n}} & =0 & & \text { on } \partial \Omega
\end{array}
$$

with $\mathbf{u}^{0} \in\left[H^{1}(\Omega)\right]^{N}$. This problem has a unique solution. Furthermore,

(i) Total mass is conserved over time. That is

$$
\int_{\Omega} \sum_{k=1}^{N} u_{k}(t) d \mu=\int_{\Omega} \sum_{k=1}^{N} u_{k}^{0}
$$

(ii) For each $t>0$, and for each $d \in \mathbb{N}$,

$$
u(t) \in H^{d}(\Omega) .
$$

(iii) The long time limit of $\mathbf{u}$ is a constant function of $x$. In fact,

$$
\left(\lim _{t \rightarrow+\infty} u(t)\right)(x)=\left(\int_{\Omega} \sum_{k=1}^{N} u_{k}^{0}\right) \mathbf{s} .
$$

Thus, the system is stable.

The spaces $H^{d}(\Omega)$ are standard Sobolev spaces of $d$ times weakly differentiable functions.

Proof. We observe that the operator induced by the matrix $A$ onto $H^{1}(\Omega)^{n}$ is bounded, and thus Lipschitz, from where existence and uniqueness follow (see for instance [3]). 
To prove 1-3, let $\left\{\varphi_{j}, \lambda_{j}\right\}_{j=0,1, \ldots, \infty}$ be the eigensystem of $-\Delta$ on the domain $\Omega$ with homogeneous Neumann boundary conditions, normalized to be an orthonormal basis of $L_{2}(\Omega)$. Recall that if these eigenvalues are sorted so that $\lambda_{k} \leq \lambda_{k+1}$, then $\lambda_{0}$ is zero, is a simple eigenvalue, and the corresponding eigenfunction is a constant. As a consequence, the integral of $\varphi_{j}$ is zero wherever $j \geq 1$. writing

The solution of (3.15) can be given explicitly in terms of this eigensystem. Indeed,

$$
\mathbf{u}^{0}=\sum_{j=0}^{+\infty} \mathbf{u}_{j} \varphi_{j}
$$

we have that

$$
\mathbf{u}(t)=\sum_{j=0}^{+\infty} e^{t\left(A-\lambda_{j} D\right)} \mathbf{u}_{j}^{0} \varphi_{j} .
$$

But the eigenvectors of $A-\lambda_{j} D$ have (again using Gerschgorin circles) real part smaller than or equal to $-\lambda_{j} D_{\min }$, where $D_{\min }$ is the smallest of all diffusion coefficients. Thus, if we write

$$
\mathbf{u}(t)=\sum_{j=0}^{+\infty} \mathbf{u}_{j}(t) \varphi_{j}
$$

we obtain the estimate

$$
\left\|\mathbf{u}_{j}(t)\right\|^{2} \leq e^{-t \lambda_{j} D_{\min }}\left\|\mathbf{u}_{j}^{0}\right\|^{2},
$$

where the norm in question is the standard Euclidean norm. Given $d$, the function $\mathbf{u}(t)$ is in $\left[H^{d}(\Omega)\right]^{N}$ whenever

$$
\sum_{j=0}^{+\infty}\left\|\mathbf{u}_{j}(t)\right\|^{2} \lambda_{j}^{d}<+\infty .
$$

For a given $m$, we have

$$
\begin{aligned}
\sum_{j=0}^{m}\left\|\mathbf{u}_{j}(t)\right\|^{2} \lambda_{j}^{d} & \leq \sum_{j=0}^{m} e^{-t \lambda_{j} D_{\min }}\left\|\mathbf{u}_{j}^{0}\right\|^{2} \\
& \leq C \sum_{j=0}^{m}\left\|\mathbf{u}_{j}^{0}\right\|^{2},
\end{aligned}
$$

where the constant $C$ depends on $d$ but not on $m$. From our choice of $\mathbf{u}^{0}$ it follows that the last expression of (3.20) converges when $m \rightarrow+\infty$. This shows (3.19) and thus proves 2 .

Another consequence of (3.18) is that if $j \geq 1$, then $\mathbf{u}_{j}(t) \rightarrow 0$ when $t \rightarrow 0$. From this, we obtain that

$$
\begin{aligned}
\int_{\Omega} \mathbf{u}(t) d \mu & =\int_{\Omega} \sum_{j=0}^{N} e^{t\left(A-\lambda_{j} D\right)} \mathbf{u}_{j}^{0} \varphi_{j} d \mu \\
& =\int_{\Omega} e^{t A} \mathbf{u}_{0}^{0} \varphi_{0} d \mu .
\end{aligned}
$$

Now, 1 and 3 follow immediately from lemma 3.5. 
3.5. Remarks on memory and initial conditions. Subdiffusive processes have memory, which means that the history of the system, and not only its current configuration, determines its future. Subdiffusion is not a Markovian process. While the future of the pseudospecies $u_{k}$ within our reaction diffusion system is, certainly, determined by their present states, and not by their past states, the situation is different for $u=\sum_{k=1}^{N} u_{k}$. Information on the past of $u$ is stored within the concentrations profiles of the pseudospecies. A full discussion of this issue would be beyond the scope of this article and shall be studied in detail elsewhere.

Reasonable initial conditions for a particular situation should be obtainable from modeling assumptions. We give two simple examples to illustrate the principle.

Returning to the RCTRW model, suppose that robots are distributed with a local concentration given by $u^{0}$. If the robots have been off, and are all turned on at once, $w_{k} u^{0}$ will end up in state $k$, as they choose it with probability $w_{k}$. For this scenario, it is reasonable to consider the initial conditions $u_{k}^{0}=w_{k} u^{0}, k=1,2, \ldots, N$. We will call this the jump-start initial conditions. Similarly, if the robots have been turned on for a long time, the distribution of robots in a particular state will be very close to the steady state distribution given by $\mathbf{s}$. In this case, the initial conditions $u_{k}^{0}=s_{k} u^{0}$ would be more appropriate. We will call them equilibrium initial conditions.

4. Choice of parameters. The generalized diffusion coefficient $K_{\alpha}$ appearing in (2.3) does not have an obvious interpretation within our reaction diffusion model. The reason is that we never rescale the PDFs going to the limit as is described in $\S$ 2.2. We could of course try the brute force approach and fit $P, Q$, and $\Sigma^{2}$ to give a certain $K_{\alpha}$ within a certain time interval using relation (2.2).

One can derive other phenomenological parameters for the reaction diffusion system (3.1) which seem more natural. Thus, for a return to normal diffusion for long time scales, we are interested in specifying this long term diffusion coefficient, and also the time of return. Another, less obvious implication of our modeling approach is the existence of an initial, or maximal, diffusion constant.

In what follows we derive expressions for these phenomenological parameters. We also show that these parameters completely specify the internal parameters $P, Q$, and $\Sigma^{2}$.

4.1. Average local diffusion coefficient. While the RCTRW was the starting point for our derivation, to impose physical behavior we will come from a different angle, treating the pseudospecies as diffusing by Brownian motion, i.e., canonically obeying Fick's laws. The reason for this is that we will have to characterize behavior for a given time instant $t$. As the model is derived from a modification of the CTRW, and we do not shrink the timescales as discussed in $\S 2.2$, we do not have a description for very short term behavior.

If we neglect the reaction term, and think of each pseudospecies as a separate substance diffusing according to Fick's laws, we can derive an average local diffusion coefficient from the local concentrations. This local diffusion coefficient describes very short term diffusive behavior.

Let $\mathbf{u}=\sum_{k=1}^{N} u_{k}$ be a solution of problem (3.15). We assume that all $u_{k}$ are positive, and define the average local diffusion coefficient by

$$
\bar{D}(x, t):=\frac{\sum_{k=1}^{N} u_{k}(x, t) D_{k}}{u(x, t)},
$$

where $D_{k}$ is the diffusion coefficient of the $k$-th pseudospecies. Note that because of theorem 3.6, 2, point evaluation is well defined for $t>0$. 
To motivate this definition, let us choose a particle that is at place $x_{0}$ at time $t_{0}$, and let $\phi\left(t-t_{0}\right)$ be its squared displacement at time $t>t_{0}$. If the particle belongs to the $k$-th pseudospecies, and we neglect the reaction term, then its mean square displacement will be given by

$$
\left\langle\phi_{k}\left(t-t_{0}\right)\right\rangle=2\left(t-t_{0}\right) D_{k},
$$

where the average is taken over all possible particle paths. The probability that it belongs to the $k$-th pseudospecies is given by

$$
\frac{u_{k}\left(x_{0}, t_{0}\right)}{u\left(x_{0}, t_{0}\right)}
$$

so that

$$
\begin{aligned}
\left\langle\phi\left(t-t_{0}\right)\right\rangle & =\sum_{k=1}^{N} \frac{u_{k}\left(x_{0}, t_{0}\right)}{u\left(x_{0}, t_{0}\right)}\left\langle\phi\left(t-t_{0}\right)\right\rangle \\
& =2\left(t-t_{0}\right) \sum_{k=1}^{N} \frac{u_{k}\left(x_{0}, t_{0}\right)}{u\left(x_{0}, t_{0}\right)} D_{k} \\
& =2\left(t-t_{0}\right) \bar{D}\left(x_{0}, t_{0}\right) .
\end{aligned}
$$

Let $u=\sum_{k=1}^{N} u_{k}$ be a solution of problem (3.15) with constant initial conditions on all $\Omega$. For simplicity, we take $u=1$. If we take jump-start initial conditions (see $\S$ $3.5)$, we obtain an initial average diffusion coefficient

$$
\bar{D}_{0}=\bar{D}(x, 0)=\sum_{k=1}^{N} w_{k} D_{k} .
$$

As $t \rightarrow+\infty$, we obtain from theorem 3.6 that $u_{k}(x, t) \rightarrow s_{k}$, so the long term average diffusion will be

$$
\bar{D}_{\infty}=\lim _{t \rightarrow+\infty} \bar{D}(x, t)=\sum_{k=1}^{N} s_{k} D_{k} .
$$

As $w_{1}<w_{2}<\cdots<w_{N}$, and $s_{1}>s_{2}>\cdots>s_{N}$, we can define a time of "essential return to normal diffusion" $\tau_{\rho}$ as the smallest $t$ for which $u_{1}(t) \geq u_{2}(t) \geq$ $\cdots \geq u_{N}(t)$ holds. It should be noted that a rigorous definition of a finite time of return to normal diffusion makes little sense, as the limit $\mathbf{u}=\mathbf{s}$ is not achieved in finite time. The word "essential" in the name of $\tau_{\rho}$ reflects the intuitive insight that the anomalous diffusivity observed later in the numerical experiments is due to the redistribution of bulk from fastly diffusing pseudospecies to slower ones. The time $\tau_{\rho}$ marks a time when this redistribution is, informally speaking, almost complete.

We will see later in the numerical results that, when the number of species is low, this choice of $\tau_{\rho}$ can cause problems, as in this case, redistribution of bulk is relatively far from being finished at this time.

4.2. Imposing physical parameters. We have, thus, three physical parameters we wish to impose on the system. Namely, the initial, or maximum diffusion $\bar{D}_{0}$, the final, or long-term diffusion $\bar{D}_{\infty}$, and the time of essential return to normal diffusion, $\tau_{\rho}$. Once we have chosen the number of pseudospecies $N$, we have three 
internal parameters left. The bounds of the interval of integration in (3.8) $(P$ and $Q)$, and the jump length variance $\Sigma^{2}$. We will now show that for each physical parameter set $0<\bar{D}_{\infty}<\bar{D}_{0}<+\infty$ and $0<\tau_{\rho}<+\infty$, there exist corresponding internal parameters $-\infty<P<Q<+\infty$, and $\Sigma^{2}>0$. It turns out that they can be obtained without too much effort.

To simplify this endeavor, we will begin by observing that the factor of reduction of the diffusion coefficient is independent of $\Sigma^{2}$. Indeed, since $D_{k}=\Sigma^{2} /\left(2 \tau_{k}\right)$, it holds that

$$
D_{\text {drop }}=\frac{\bar{D}_{\infty}}{\bar{D}_{0}}=\frac{\sum_{k=1}^{N} \frac{s_{k}}{\tau_{k}}}{\sum_{k=1}^{N} \frac{w_{k}}{\tau_{k}}} .
$$

Thus, we can try to manipulate $P$ and $Q$ to find the desired $D_{\text {drop }}$ and $\tau_{\rho}$, and obtain $\Sigma^{2}$ afterward from a simple algebraic expression.

From $\S 3.3$, and from lemma 3.4 we have algebraic expressions for $s_{k}, w_{k}$, and $\tau_{k}$ in terms of the quadrature nodes $x_{k}$, which in turn can be expressed in terms of $A$ and $h$. We fix $N$ and write

$$
\Phi(A, h):=D_{\mathrm{drop}}(A, h) .
$$

LEMmA 4.1. The function $\Phi(A, h)$ depends only on $h$. In fact, writing $\Phi(h)=$ $\Phi(A, h)$ we have

$$
\begin{gathered}
\Phi(h)=\frac{\sum_{k=1}^{N} e^{h(\alpha+1) k} \sum_{k=1}^{N} e^{h(1-\alpha) k}}{\sum_{k=1}^{N} e^{h[(1-\alpha)(N-k+1)+k]} \sum_{k=1}^{N} e^{h \alpha k}} \\
=\frac{e^{h(1-\alpha)(1+N)} \theta(h, \alpha)^{2}}{\theta(h, \alpha+1) \theta(h, 1-\alpha)}
\end{gathered}
$$

with

$$
\theta(h, \kappa):=\sum_{k=1}^{N}\left(e^{h \kappa}\right)^{k}=\frac{e^{h \kappa}-e^{h \kappa(N+1)}}{1-e^{h \kappa}} .
$$

The proof is a straightforward sequence of algebraic manipulations and is thus omitted.

Lemma 4.2. For each $D_{\text {drop }} \in(0,1)$ there exists* $h>0$ such that $\Phi(h)=D_{\text {drop }}$.

Proof. The function $\Phi(h)$ is continuous for $h>0$. If $h \rightarrow 0^{+}$, we observe that $\Phi(h) \rightarrow 1$. The proof is finished if we can show that

$$
\lim _{h \rightarrow+\infty} \Phi(h)=0 .
$$

Indeed, using that $\sum_{k=1}^{N} r^{k} \leq C r^{k+1}$ for fixed $r>1$, for some $C>0$ and for all $N \geq 1$, we obtain that

$$
\begin{aligned}
0<\Phi(h) & \leq C \frac{e^{h(\alpha+1)(N+1)} e^{h(1-\alpha)(N+1)}}{\sum_{k=1}^{N} e^{h[(1-\alpha)(N-k+1)+k]} \sum_{k=1}^{N} e^{h \alpha k}} \\
& \leq C \frac{e^{h(\alpha+1)(N+1)} e^{h(1-\alpha)(N+1)}}{e^{N h} e^{h(1-\alpha) N} e^{h}} \leq \frac{C}{e^{h(1-\alpha) N}},
\end{aligned}
$$

${ }^{*}$ We were not able to prove that this $h$ is unique, although we certainly believe it is. The difficulty is that it is not clear how to prove that $\Phi$ is a strictly monotone decreasing function. Our attempt to show that $\Phi^{\prime}(h)<0$ for $h>0$ encountered their obstacle in the rather convoluted expression for $\Phi^{\prime}$. 
which confirms (4.3) and thus finishes the proof.

Given $P<Q$, let us write $A[P, Q]$ for the reaction matrix constructed according to $(3.3),(3.4),(3.10)$, and (3.9). We now define

$$
\tau_{\rho}[P, Q]=\inf \left\{t: \mathbf{v}=\left(v_{k}\right)=e^{t A[P, Q]} \text { satisfies } v_{i-1} \geq v_{i}, i=2,3, \ldots, N\right\} .
$$

LEMmA 4.3. It holds that $\tau_{\rho}[P+\delta, Q+\delta]=e^{-\delta} \tau_{\rho}[P, Q]$.

The proof of this lemma follows from observing that $A[P+\delta, Q+\delta]=e^{-\delta} A[P, Q]$, and applying this knowledge to (4.4).

In sum, to set up the system, we start by finding $h$ such that $\Phi(h)=D_{\text {drop }}$, which can be done using Newton's method. Then, we set

$$
P=-\log \left(\frac{\tau_{\rho}}{\tau_{\rho}[0, N h]}\right), \quad Q=P+N h,
$$

and finally

$$
\Sigma^{2}=\frac{2 \bar{D}_{0}}{\sum_{k=1}^{N} \frac{w_{k}}{\tau_{k}}}
$$

With these parameters, the reaction diffusion system is completely specified.

REMARK 4.4. For the numerical computation of $P$ and $Q$, it is helpful if the procedure summarized in (4.5) is modified to read

$$
P=-P_{S}-\log \left(\frac{\tau_{\rho}}{\tau_{\rho}\left[P_{S}, P_{S}+N h\right]}\right), \quad Q=P+N h .
$$

Mathematically, any choice of $P_{S}$ leads to the same $P$ and $Q$, but numerically, $P_{S}$ can be used to avoid overflows or underflows in $\tau_{\rho}\left[P_{S}, P_{S}+N h\right]$. In our experiments, we used $P_{S}=10-N h$.

5. Numerical experiments. In this section, we would like to test the system we have set up so far. We will start by checking how well the systems we obtain behave in the benchmark set up at the beginning of $\S 3$. We will also produce a few pictures of solutions and compare their shape with solutions of the subdiffusive fractional diffusion equation. Finally, we will include a sequence showing the effects of return to Fickian diffusion.

For simplicity, we will discretize (3.1) in space using second order, centered finite differences, and integrate the resulting system of ordinary differential equations in time using the implicit Euler formula. The resulting linear systems of equations are solved using Gaussian elimination, which is fast and stable for the associated tridiagonal matrices.

Our domain will be the whole real line. As the initial condition we approximate the Dirac delta (at zero) by a very slim and high hat function. While the support of the continuous solution is infinite for all $t$, it decays rather fast with $|x|$, and thus we track the solution by considering a sequence of growing domains. Whenever a domain threatens to become too small, we increase its length by a fixed factor, and interpolate the solution on the previous domain to the new grid after extending it by zero.

In more concrete terms, the initial condition is given by $u_{k}^{0}=w_{k} u^{0}$, with

$$
u^{0}(x)= \begin{cases}10^{4}+x 10^{8} & \text { if } x \in\left(-10^{-4}, 0\right] \\ 10^{4}-x 10^{8} & \text { if } x \in\left(0,10^{-4},\right. \text { and } \\ 0 & \text { otherwise. }\end{cases}
$$


We solve this problem numerically, using 1024 knots on the domain of interest. We start the computation on $I_{0}=(-0.025,0.025)$, and define $I_{k+1}=(1+0.25) I_{k}$. We solve in $I_{k}$ until the solution at the endpoints exceed a factor of $10^{-12}$ times the maximum of the solution on the domain. Then, we extend the solution by zero, and use it as the initial condition for the same problem on the domain $I_{k+1}$.

We integrate from $t=0$ until $t=10^{5}$. The size of the time step is chosen using the formula

$$
h_{t}=10^{-7}+t \cdot 5 \cdot 10^{-3} .
$$

The parameters are as follows. We take $\alpha=0.5, \bar{D}_{0}=10, \bar{D}_{\infty}=0.01$, and $\tau_{\rho}=100$. With $N=16$ we obtain the integration interval $P_{16}=-5.67, Q_{16}=10.51$, and the jump length variance $\Sigma_{16}^{2}=1.78 \cdot 10^{-3}$. Values for corresponding $w_{k}, \tau_{k}$, and $D_{k}$ can be found in table 5.1. With $N=4$ we obtain $P_{4}=-9.611, Q_{4}=9.078$, and $\Sigma_{4}^{2}=2.61 \cdot 10^{-2}$.

\begin{tabular}{|l|l|l|l|l|}
\hline$k$ & $D_{k}$ & $\tau_{k}$ & $w_{k}$ & $s_{k}$ \\
\hline \hline 1 & $5.086 \mathrm{e}-06$ & $1.750 \mathrm{e}+02$ & $2.019 \mathrm{e}-04$ & $3.970 \mathrm{e}-01$ \\
2 & $1.398 \mathrm{e}-05$ & $6.367 \mathrm{e}+01$ & $3.347 \mathrm{e}-04$ & $2.394 \mathrm{e}-01$ \\
3 & $3.843 \mathrm{e}-05$ & $2.316 \mathrm{e}+01$ & $5.550 \mathrm{e}-04$ & $1.444 \mathrm{e}-01$ \\
4 & $1.056 \mathrm{e}-04$ & $8.426 \mathrm{e}+00$ & $9.201 \mathrm{e}-04$ & $8.710 \mathrm{e}-02$ \\
5 & $2.904 \mathrm{e}-04$ & $3.065 \mathrm{e}+00$ & $1.526 \mathrm{e}-03$ & $5.254 \mathrm{e}-02$ \\
6 & $7.982 \mathrm{e}-04$ & $1.115 \mathrm{e}+00$ & $2.529 \mathrm{e}-03$ & $3.169 \mathrm{e}-02$ \\
7 & $2.194 \mathrm{e}-03$ & $4.057 \mathrm{e}-01$ & $4.193 \mathrm{e}-03$ & $1.911 \mathrm{e}-02$ \\
8 & $6.031 \mathrm{e}-03$ & $1.476 \mathrm{e}-01$ & $6.953 \mathrm{e}-03$ & $1.153 \mathrm{e}-02$ \\
9 & $1.658 \mathrm{e}-02$ & $5.369 \mathrm{e}-02$ & $1.153 \mathrm{e}-02$ & $6.953 \mathrm{e}-03$ \\
10 & $4.558 \mathrm{e}-02$ & $1.953 \mathrm{e}-02$ & $1.911 \mathrm{e}-02$ & $4.193 \mathrm{e}-03$ \\
11 & $1.253 \mathrm{e}-01$ & $7.105 \mathrm{e}-03$ & $3.169 \mathrm{e}-02$ & $2.529 \mathrm{e}-03$ \\
12 & $3.444 \mathrm{e}-01$ & $2.585 \mathrm{e}-03$ & $5.254 \mathrm{e}-02$ & $1.526 \mathrm{e}-03$ \\
13 & $9.467 \mathrm{e}-01$ & $9.403 \mathrm{e}-04$ & $8.710 \mathrm{e}-02$ & $9.201 \mathrm{e}-04$ \\
14 & $2.602 \mathrm{e}+00$ & $3.421 \mathrm{e}-04$ & $1.444 \mathrm{e}-01$ & $5.550 \mathrm{e}-04$ \\
15 & $7.153 \mathrm{e}+00$ & $1.244 \mathrm{e}-04$ & $2.394 \mathrm{e}-01$ & $3.347 \mathrm{e}-04$ \\
16 & $1.966 \mathrm{e}+01$ & $4.527 \mathrm{e}-05$ & $3.970 \mathrm{e}-01$ & $2.019 \mathrm{e}-04$ \\
\hline \multicolumn{4}{|c}{ TABLE 5.1} \\
\hline \multicolumn{4}{|c}{5}
\end{tabular}

Parameter values, example with 16 pseudospecies

5.1. Diffusive behavior. If $X(t)$ denotes the position of a particle that was at $x_{0}=0$ at time $t=0$, and the probability distribution for $X(t)$ is given by $u(x, t)$ with $u(x, t)=u(-x, t)$, then the mean square displacement of the particle is given by

$$
\left\langle X(t)^{2}\right\rangle=2 \int_{0}^{+\infty} y^{2} u(y, t) d y .
$$

Since our initial condition was chosen to approximate $\delta_{0}$, we will interpret the solution obtained from the simulation in this manner. In figure 5.1 we show plots of $\left\langle x(t)^{2}\right\rangle /\left(t^{\alpha}\right)$ (which is constant for pure subdiffusion with parameter $\alpha=0.5$ ) and $\left\langle x(t)^{2}\right\rangle /(2 t)$, which is constant for normal diffusion, and reflects the current diffusion coefficient.

We observe that for $t$ approximately between $10^{-4}$ and $10^{2}$, propagation is indeed subdiffusive. Note also the return to normal diffusion for larger times.

Subdiffusive behavior is still observed for $N=4$, although the details are somewhat different. See figure 5.3. The longer extent of the timescales with subdiffusive 


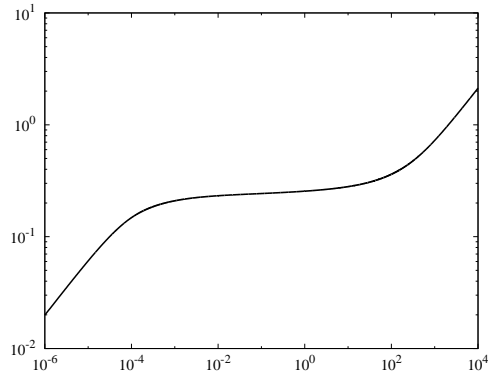

(a) Behavior of $\frac{\left\langle x(t)^{2}\right\rangle}{t^{\alpha}}$

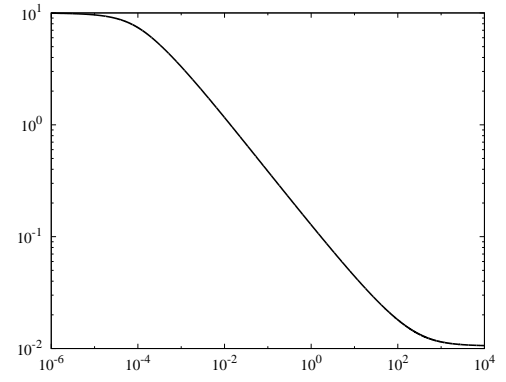

(b) Behavior of $\frac{\left\langle x(t)^{2}\right\rangle}{2 t}$

FIG. 5.1. Diffusive behavior of the reaction diffusion model

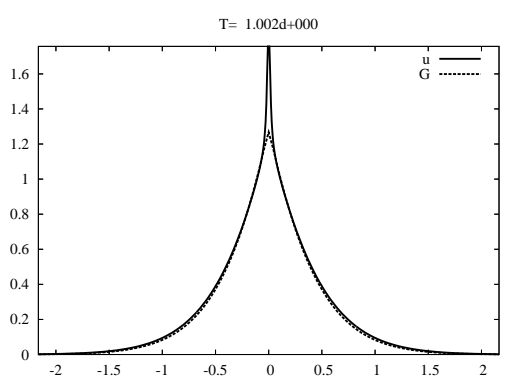

(a) Solution $u$ at time $t=1$, together with a plot of the fundamental solution $G$.

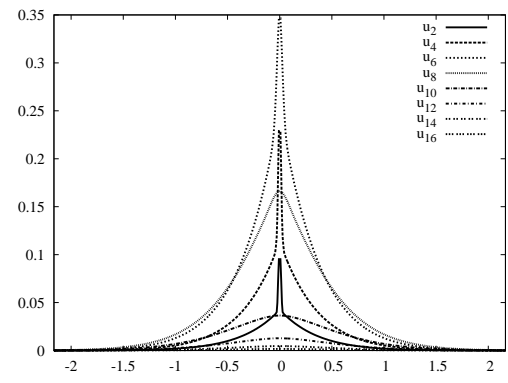

(b) Pseudospecies $u_{k}$ for $k$ even

FIG. 5.2. Solution and pseudospecies profiles

behavior is due to the fact that the condition we have chosen to define the time of essential return to normal diffusion (see $\S 4.1$ ) is not adequate if only a few species are present.

5.2. Solution shape. The solution of the subdiffusive fractional diffusion equation with such an initial condition has a characteristic cusp shape. We can obtain a fairly similar shape if we have enough pseudospecies; see figure 5.2. The reference is the fundamental solution, or Green function, of the subdiffusive FDE,

$$
G(x, t)=\frac{1}{2 \sqrt{D_{\alpha}} t^{\alpha / 2}} M\left(\frac{|x|}{2 \sqrt{D_{\alpha}} t^{\alpha / 2}}, \frac{\alpha}{2}\right),
$$

with

$$
M(z, \nu)=\frac{1}{\pi} \sum_{n=1}^{+\infty} \frac{(-z)^{n-1}}{(n-1) !} \Gamma(n \nu) \sin (n \nu \pi)
$$

see [7] for details. In figure 5.2(a), the parameters for the fundamental solution are $\alpha=0.5, D_{\alpha}=0.1$, and $t=1$.

In figure 5.3 we observe that the profile with $N=4$ is somewhat different.

5.3. Return to normal diffusion. Finally, we show in figure 5.4 a sequence of plots of the solution on its way through the subdiffusive regime towards a return to normal diffusion. 


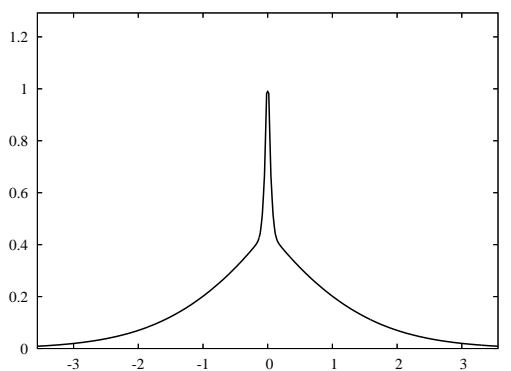

(a) Solution $u$ (with $N=4$ ) at time $t=1$.

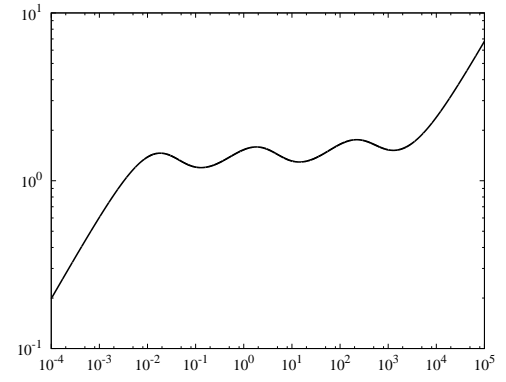

(b) Behavior of $\frac{\left\langle x(t)^{2}\right\rangle}{t^{\alpha}}$ for $N=4$.

FIG. 5.3. Solution and pseudospecies profiles
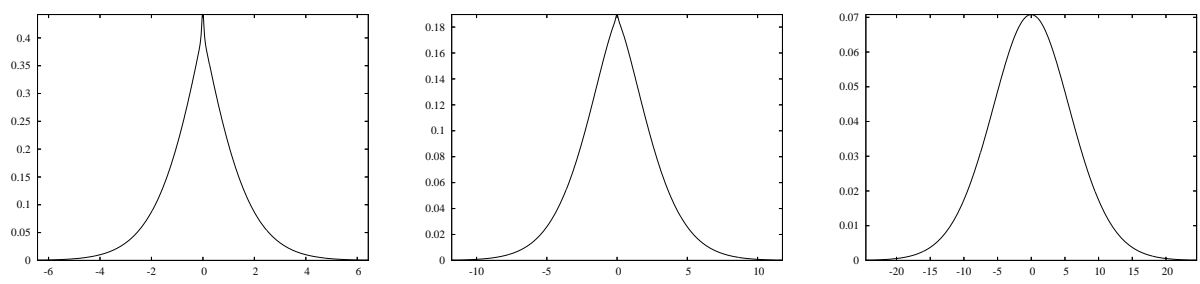

FIG. 5.4. Solutions for $t=4.835 \cdot 10^{1}, t=2.985 \cdot 10^{2}$, and $t=1.836 \cdot 10^{3}$.

Acknowledgments. We thank Jürgen Warnatz and the Reactive Flows group at the IWR in Heidelberg for their kind hospitality. We further thank Christophe Rhodes for interesting remarks. All remaining mistakes are the authors sole responsibility.

\section{REFERENCES}

[1] G. Beylkin and L. Monzón, On approximation of functions by exponential sums, Appl. Comput. Harmon. Anal., 19 (2005), pp. 17-48.

[2] J. P. Bouchaud and A. Georges, Anomalous diffusion in disordered media: Statistical mechanisms, models and physical applications, Physics Reports, 195 (1990), pp. 127-293.

[3] I. D. Chueshov, Introduction to the Theory of Infinite-Dimensional Dissipative Systems, EMIS Electronic Library of Mathematics, 2002.

[4] Gene H. Golub and Charles F. van Loan, Matrix Computations, North Oxford Academic, third ed., 1996.

[5] R. Gorenflo and F. Mainard, Fractional diffusion processes: Probability distributions and continuous time random walk, in Long Range Dependent Processes: Theory and Applications, G. Rangarajan and M. Ding, eds., Springer, Heidelberg, 2003, pp. 148-166.

[6] K. D. Lewandowska And T. Kosztołowicz, Numerical study of subdiffusion equation, tech. report, arXiv, 2006.

[7] F. Mainardi, P. Paradisi, and R. Gorenflo, Probability distributions generated by fractional diffusion equations, in Econophysics: An Emerging Science (Proceedings of the first Econophysics workshop, Budapest 1997), J. Kertész and I. Kondor, eds., Published online, 2002.

[8] R. Metzler And J. Klafter, The random walk's guide to anomalous diffusion: A fractional dynamics approach, Physics Reports, 339 (2000), pp. 1-77.

[9] E. W. Montroll and G. H. Weiss, Random walks on lattices. ii, Journal of Mathematical Physics, 6 (1965), pp. 167-181.

[10] M. J. SAxton, Chemically limited reactions on a percolation cluster, J. Phys. Chem., 116, pp. 203-208.

[11] — A biological interpretation of transient anomalous diffusion. i. qualitative model, Biophysical Journal, 92 (2007), pp. 1178-1191. 
[12] M. Weiss, M. Elsner, F. Kartberg, And T. Nilsson, Anomalous subdiffusion is a measure for cytoplasmic crowding in living cells, Biophysical Journal, 87 (2004), pp. 3518-3524. 